\title{
Antibiotic Stewardship in Orthopädie und Unfallchirurgie
}

Dagmar Horn, Robin Köck, Dirk Wähnert, Steffen Roßlenbroich, Michael Raschke

\section{Einleitung}

Die adäquate Prävention und Therapie von Infektionen nimmt einen immer größer werdenden Platz in der Orthopädie und Unfallchirurgie ein. Dabei hat sich die Rate nosokomialer Infektionen in deutschen Krankenhäusern in den letzten Jahren kaum verändert und beträgt nach wie vor ca. 5\%. Postoperative Wundinfektionen stellen mit einer Prävalenz von 1,31 pro 100 Fälle die häufigsten nosokomialen Infektionen (24\% aller Infektionen) dar [1]. Während das Risiko einer Infektion bei geschlossenen Frakturen oder bei Implantation von Hüft- bzw. Kniegelenksprothesen mit ca. 1-5\% verhältnismäßig niedrig ist, steigt es insbesondere bei Revisionseingriffen oder nach offenen Frakturen auf bis zu 30\% [2]. Wesentliche Risikofaktoren für eine postoperative Wundinfektion sind unter anderem hohes Alter, Adipositas und Begleiterkrankungen wie z. B. Diabetes mellitus [3-5]. Bei steigender Lebenserwartung ist zukünftig mit einer weiteren Zunahme von Infektionen zu rechnen.

Die Kombination aus chirurgischer und antibiotischer Therapie führt in etwa 70-90\% zu einer erfolgreichen Behandlung der Infektion [6, 7], was in Anbetracht einer stetig verbesserten Operationstechnik unbefriedigend ist. Hinzu kommt die Zunahme von Infektionen durch antibiotikaresistente Erreger. Zu diesen zählen insbesondere Chinolon- und Carbapenemresistenzen sowie Resistenzen gegenüber Piperacillin/Tazobactam und Cephalosporinen der 3. Generation bei gramnegativen Erregern sowie Clostridium difficile. Hierdurch werden zum einen die Therapieoptionen reduziert und zum anderen Morbidität, Letalität und Behandlungskosten erhöht [1]. Eine Entwicklung von neuen antibiotischen Substanzen, die adäquate therapeutische Optionen gegen diese Erreger dauerhaft sichern, ist dagegen kaum zu erwarten. Erschwerend kommt hinzu, dass entsprechend einer aktuellen Untersuchung etwa die Hälfte (49\%) der Antibiotikaverordnungen in chirurgischen Abteilungen als inadäquat zu bewerten ist [8]. Zu Fehlern kommt es hier insbesondere bei der Indikationsstellung zur Antibiotikatherapie, aber auch bei der Auswahl des Antibiotikums, seiner Dosierung und der Therapiedauer. Die Gründe hierfür sind vielfältig ( $\bullet$ Tab. 1$)$.
Merke

Vor diesem Hintergrund ist es von entscheidender Bedeutung, die Prävention und Diagnostik von Infektionen sowie die Anwendung von Antibiotika in Orthopädie und Unfallchirurgie zu optimieren, um die Inzidenz von Infektionen zu senken, die Therapieerfolgsrate zu erhöhen und der zunehmenden Resistenzentwicklung entgegenzuwirken.

Eine mögliche Strategie, dieses Ziel zu erreichen, ist die Implementierung von sogenannten Antibiotic-Stewardship-Maßnahmen (ABS-Maßnahmen) in Orthopädie und Unfallchirurgie. Obwohl diese Maßnahmen bereits seit 1998 von der Europäischen Union (EU) sowie durch Änderungen des Infektionsschutzgesetzes (2011) und Veröffentlichung einer S3-Leitlinie zu „Strategien zur Sicherung rationaler Antibiotika-Anwendung im Krankenhaus“ (2013) in Deutschland empfohlen wurden, findet bis heute eine konsequente Umsetzung in deutschen Krankenhäusern nicht flächendeckend statt.

Unter ABS sollte dabei nicht nur ein Leitfaden zur Optimierung oder gar Reduktion von „Antibiotikagaben“ verstanden werden, sondern ein Gesamtkonzept aus Maßnahmen zu „Antibiotic Stewardship“, „Infection Control Stewardship“ und „Diagnostic Stewardship“, das niederländische Kollegen deshalb auch als „AID Stewardship“ bezeichnen [9]. Dieser multimodale Ansatz ist besonders in der Orthopädie und Unfallchirurgie von entscheiden-

- Tab. 1 Gründe für inadäquaten Gebrauch von Antibiotika.

- Unsicherheit bei der Diagnose einer Infektion bzw. inadäquate Durchführung mikrobiologischer Diagnostik

- unzureichende Fortbildung und Erfahrung bei der Behandlung von Infektionen

- unzureichende Kenntnisse zur lokalen Epidemiologie von Antibiotikaresistenzen

- Fehlinterpretation mikrobiologischer und (histo-)pathologischer Befunde

- unzureichende Anleitung und Unterstützung bei der Behandlung von Infektionen bzw. durch fehlende oder schlecht etablierte lokale Therapieleitlinien

- Fehldosierung von Antibiotika (insbesondere Unter- und Überdosierung)

- keine Möglichkeiten zum therapeutischen Drug Monitoring einer Antibiotikatherapie (z. B. Spiegelbestimmung) 
der Bedeutung. Wir wissen heute, dass $>50 \%$ der in der muskuloskeletalen Chirurgie vorkommenden Infektionen als endogene Infektionen (von der eigenen mikrobiellen Flora eines Patienten ausgehend) zu bewerten sind. Insofern ist es im Sinne der Prävention und modernen Krankenhaushygiene („Infection Control Stewardship“) auch entscheidend, dass durch eine optimale Infektionsdiagnostik („Diagnostic Stewardship“) Infektionen frühzeitig erkannt und gezielt behandelt werden (= sekundäre Prävention). Gleichzeitig sind auch die primärpräventiven Infektionspräventionsansätze in der Orthopädie und Unfallchirurgie (z. B. perioperative Antisepsis, perioperative Antibiotikaprophylaxe) wichtige Bausteine in einem „AID Stewardship“-Konzept, das von „klassischen“ Präventionskonzepten (Hygienepläne zur Wundversorgung, Sterilgutaufbereitung etc.) zu flankieren ist.

\section{Antibiotic-Stewardship-Strukturen}

Unter dem Begriff „Antibiotic Stewardship“ (ABS) (im Sinne der oben ausgeführten „AID Stewardship“-Definition) wird ein Bündel von Maßnahmen zur Verbesserung der Antibiotikatherapie mit dem Ziel, die Verordnungsqualität und das Outcome von Patienten zu verbessern, zusammengefasst. Weitere Ziele sind die Eindämmung der mikrobiellen Resistenzentwicklung durch Senkung von Selektionsdruck durch inadäquate Antibiotikaanwendung und die Vermeidung antibiotikaassoziierter Folgeerkrankungen (C.-difficile-assoziierte Diarrhö) zu verlangsamen bzw. zu stoppen. Infektiologische Fragestellungen des klinischen Alltags sollten im Rahmen von ABS durch ein interdisziplinäres Team adressiert werden. Hierzu gehören neben der Therapieoptimierung bei konkreten, individuellen Fallkonstellationen vor allem auch die Erarbeitung, Implementierung und (Re-)Evaluation lokaler Diagnostik- und Therapiealgorithmen bzw. -standards.

Eine Reihe klinischer Untersuchungen konnte zeigen, dass ABS das Outcome der Patienten verbessert und es gleichzeitig zu weniger antibiotikaassoziierten Nebenwirkungen, weniger infektionsbedingten Wiederaufnahmen von Patienten und einer reduzierten bakteriellen Resistenzentwicklung führt [8].

Auch wenn ABS nicht das primäre Ziel einer Kostenersparnis hat, so führte eine optimierte Antibiotikatherapie in vielen Fällen auch zu einer Reduktion der Antibiotikakosten $[10,11]$. Hierzu gehören neben den reinen Kosten für die eingesparten Antibiotika auch Material- (z. B. Kosten für Infusionsbesteck und Lösungsmittel) und Personalkosten (Zeitaufwand der Pflegekraft für die Zubereitung und Applikation des Antibiotikums). Auch eine Reduktion von Infektionen durch C. difficile kann zu einer deutlichen Kostenersparnis führen (z. B. durch weniger Erlösverluste aufgrund einer Verlängerung des Krankenhausaufenthalts und durch Bettensperrungen) [12].
So kann z. B. eine konsequente Umsetzung einer Verfahrensanweisung der perioperativen Antibiotikaprophylaxe (PAP) und das damit verbundene Vermeiden einer Antibiotikaapplikation über das OP-Ende hinaus schon zu einer deutlichen Reduktion des Antibiotikaverbrauchs führen.

Um diesen Ansprüchen gerecht zu werden, besteht ein ABS-Team aus mehreren Personen mit unterschiedlichen fachlichen Qualifikationen. Hierbei handelt es sich regelmäßig um:

- infektiologisch qualifizierte Kliniker

- klinische Pharmazeuten

- Mikrobiologen oder Hygieniker

Die unterschiedlichen Expertisen dieses interdisziplinären Teams sind für die Beratung und für das Monitoring der Ergebnisse sowie für das Feedback an die direkt therapierenden Ärzte von entscheidender Bedeutung.

Die Zusammenstellung eines ABS-Teams ist in Deutschland derzeit vielerorts sicher schwieriger als in anderen Ländern. So gab es in einer aktuellen Erhebung in 1980 deutschen Krankenhäusern nur etwa 300 Ärzte mit abgeschlossener Weiterbildung „Infektiologie“ im stationären Bereich [13]. Ebenso ausgeprägt ist der Mangel an Fachärzten in den Gebieten Krankenhaushygiene (ca. 100 Fachärzte) und Mikrobiologie (ca. 300 Fachärzte) [13] und in der klinischen Pharmazie. Dass hier eine Zunahme erforderlich ist, zeigen Untersuchungen von Forsblom et al. [14]: Die Überlebenswahrscheinlichkeit von Patienten mit einer Bakteriämie durch Staphylococcus aureus ( $n=300)$ war von der Art der infektiologischen Konsultation abhängig. Während eine rein telefonische Konsultation (im Vergleich zu keinerlei infektiologischer Konsultation) das klinische Outcome der Patienten nicht verbessern konnte, hatten Patienten, bei denen eine bettseitige Therapieempfehlung erfolgte, eine signifikant erhöhte Überlebenswahrscheinlichkeit. Somit ist es von entscheidender Bedeutung, interdisziplinäres, infektiologisches Fachwissen „an das Patientenbett“ zu holen.

\footnotetext{
Merke

Die 3 zentralen Aufgabenbereiche eines adäquaten Infektionsmanagements in Orthopädie und Unfallchirurgie stellen Infektionsprävention, -diagnostik und -therapie dar.
}

Als Ergänzung zu der fachlichen Qualifikation innerhalb eines ABS-Teams werden Zusatzqualifikationen angeboten, die auch Ärzten anderer Fachdisziplinen offenstehen und in denen die Planung und Umsetzung von ABS-Maßnahmen vermittelt wird (z.B. „ABS-Experte“ der Deutschen Gesellschaft für Infektiologie [DGI]). Dies kann gerade in operativen Abteilungen sehr gut durch einen entsprechend fortgebildeten und interessierten Chirurgen erfolgen. Im Gegensatz zu anderen Fachrichtungen 
verfügt der Chirurg über besondere Kenntnisse bei der Beurteilung von Wunden und operativen Möglichkeiten zur Infektionssanierung (z.B. Belassen oder Entfernung von Osteosynthesematerial), sodass die chirurgische Expertise wertvoller Bestandteil von Visite des ABS-Teams am Patientenbett sein kann. Auf diese Weise lassen sich Kommunikationsbarrieren vermeiden und das ABS-Team wird leichter als Teil des Behandlungsteams akzeptiert und weniger als externe „Kontrollinstanz“ wahrgenommen.

Merke

Gerade im operativen Bereich ist die aktive Einbindung eines Chirurgen in das ABS-Team von entscheidender Bedeutung.

PRAXISTIPP 1

Erste Schritte zur Implementierung einer ABS-Struktur können schon das Benennen eines verantwortlichen Chirurgen und die Etablierung einer regelmäßigen interdisziplinären Besprechung oder Visite zusammen mit klinischen Pharmazeuten und Mikrobiologen sein.

Einige Punkte seien in Folge hierzu vertiefend dargestellt.

\section{Prävention}

\section{Perioperative Antibiotikaprophylaxe (PAP)}

Eine der wichtigsten Maßnahmen zur Infektionsprävention in Orthopädie und Unfallchirurgie stellt eine adäquate perioperative Antibiotikaprophylaxe (PAP) dar, da sie das Risiko einer postoperativen Wundinfektion signifikant verringern kann. Sie wird in der Regel als „Single Shot" verabreicht. Hierbei ist entscheidend, dass eine geeignete Substanz rechtzeitig vor Operationsbeginn appliziert wird, um eine optimale Verteilung und eine adäquate Konzentration in den Zielgeweben zu ermöglichen. Um dies möglichst konstant in die tägliche Routine zu integrieren, ist es zwingend erforderlich, dass das ABSTeam neben den Operateuren selbst auch die Anästhesisten, die in der Regel die perioperative Prophylaxe applizieren, miteinbezieht.

Eine Fortführung der Antibiotikaapplikation über den Zeitraum der OP hinaus zeigt in zahlreichen Studien keinen Vorteil bei der Reduktion von postoperativen Infektionen und sollte vermieden werden, da sie zu Kollateralschäden führt (z.B. Antibiotikaresistenz, C.-difficile-Infektionen, unerwünschte Arzneimittelwirkungen, gefäßkatheterassoziierte nosokomiale Infektionen). Eine Ausnahme hiervon stellen offene Frakturen dar: Aufgrund der hohen Inzidenz posttraumatischer Infektionen wird eine präemptive Therapie über den Zeitpunkt der Operation hi- naus für 24 bzw. 72 Stunden in Abhängigkeit von der Defektgröße empfohlen $[15,16]$.

Im muskuloskeletalen Bereich stellen Cephalosporine der 1. oder 2. Generation wie z. B. Cefazolin oder Cefuroxim geeignete Substanzen für die PAP dar. Sie sollten 30-60 Minuten vor OP-Beginn und gewichtsadjustiert appliziert werden. Eine perioperative Wiederholung der Applikation ist nach Ablauf von 2 Halbwertszeiten des verwandten Antibiotikums bzw. bei hohen intraoperativen Blutverlusten erforderlich. In unserer Klinik wird Cefuroxim nach 2 Stunden OP-Dauer erneut verabreicht. Dies ist Bestandteil des gemeinsamen, mit den anästhesiologischen Fachkollegen erstellten perioperativen Managementkonzepts.

Aktuell erfolgt jede 5. Antibiotikaapplikation (20\%) in deutschen Krankenhäusern als Prophylaxe in chirurgischen Abteilungen. Eine evidenzbasierte Umsetzung der Empfehlungen zur PAP findet jedoch immer noch nicht regelmäßig statt: In 3 von 4 Fällen (75\%) wird die PAP nach wie vor über den OP-Zeitraum hinaus verlängert und bleibt nur bei einem Viertel der Fälle (25\%) auf die perioperative Anwendung beschränkt.

Merke

In chirurgischen Fachabteilungen kommt es nach wie vor häufig zu einer nicht evidenzbasierten Verlängerung der perioperativen Antibiotikaprophylaxe (PAP).

Für ein ABS-Team eröffnen sich in Zusammenhang mit der PAP gleich mehrere Ansatzpunkte:

In erster Linie sollte es sich darum bemühen, dass gemeinsam mit den behandelnden Ärzten eine entsprechende Verfahrensanweisung abteilungs- und klinikübergreifend zur PAP erstellt und allen Behandlern bekannt gemacht wird. Hierzu eignen sich insbesondere regelmäßige interdisziplinäre Fortbildungsveranstaltungen für die einzelnen operativen Abteilungen, in denen die zugrunde liegende Evidenz dargestellt und auftretende Fragen oder aktuelle Fälle diskutiert werden können. In festgelegten Abständen sollte zudem überprüft werden, ob und in welchem Umfang die bestehende Verfahrensanweisung umgesetzt wurde. Hierzu gehört neben einer Überprüfung der postoperativen Anweisungen des Operateurs hinsichtlich einer möglichen Fortführung der Antibiotikaapplikation über die OP-Dauer hinaus auch die Erhebung der Einhaltung der Dosis und des optimalen Applikationszeitpunkts präoperativ und einer Wiederholung der Applikation bei längeren Eingriffen. Dies kann z.B. durch Auswertung einer definierten Stichprobe von Operationsprotokollen erfolgen, in welcher die Antibiotikagabe durch den Anästhesisten vermerkt wurde (z. B. Operationsprotokolle einer Klinik von allen Eingriffen einer festgelegten Woche pro Quartal), die nach Bewertung durch das ABS-Team mit allen beteiligten Abteilungen besprochen wird. 
In der Klinik für Unfall-, Hand- und Wiederherstellungschirurgie des Universitätsklinikums Münster konnte durch konsequente Umsetzung der Verfahrensanweisung zur PAP zusammen mit der Etablierung eines interdisziplinären Therapieansatzes (tägliche Reevaluation der Antibiotikatherapie mit klinischen Pharmazeuten und regelmäßige interdisziplinäre infektiologische Fallbesprechungen zusammen mit medizinischen Mikrobiologen) eine Vielzahl von Antibiotikaapplikationen eingespart werden. Hierdurch kam es zu einer Reduktion der Antibiotikakosten um ca. $15 \%$, ohne dass es zu einem Anstieg der periinterventionellen Infektionsrate kam [17]. Dies führte langfristig auch zu einer deutlichen Reduktion von Infektionen durch C. difficile (Inzidenz 2011: 0,46 Fälle/1000 Patiententage - Inzidenz 2013: 0,15 Fälle/1000 Patiententage) [17].

\section{PRAXISTIPP 2}

Eine regelmäßige (z. B. jährliche) Evaluation der PAP nach den Gesichtspunkten „rechtzeitige Antibiotikaapplikation vor OP-Beginn“, „rechtzeitige intraoperative Wiederholung“ und „Vermeiden einer prolongierten Gabe über das OP-Ende hinaus “ kann bereits zu einer deutlichen Reduktion des Antibiotikaverbrauchs und der Inzidenz für Infektionen durch C. difficile führen.

\section{Infektionsdiagnostik}

Ein entscheidender Schritt, um Antibiotikatherapien zu optimieren, ist die Durchführung adäquater Infektionsdiagnostik bzw. mikrobiologischer Diagnostik. Sie ist die Voraussetzung dafür, zum einen Infektionen von nicht antibiotisch zu behandelnden Erkrankungen (z. B. mechanisch bedingte Prothesenlockerung) abzugrenzen, zum anderen legt sie die Grundlage für eine Adaptation kalkuliert begonnener Antiinfektivatherapie. Letzteres ist entscheidend, um 1. durch gezielte Therapie eines nachgewiesenen Erregers das Outcome von Patienten zu verbessern und um 2. kalkuliert „breit“ wirksame Antibiotikatherapien ggf. auf empfindlich getestete Präparate mit geringerem Kollateralschaden umzustellen. Ein gutes Beispiel hierfür ist die Therapie schwerer Staphylococcusaureus-Infektionen, für die bei Einsatz unterschiedlicher, empfindlich getesteter $\beta$-Lactam-Antibiotika (Flucloxacillin vs. Cefazolin vs. Cefuroxim) deutliche Unterschiede in der klinischen Erfolgsrate dokumentiert wurden.

Ein besonderes Augenmerk für ABS-Teams ist deshalb insbesondere auch auf die Entwicklung von diagnostischen Algorithmen zu richten, da diese gerade unerfahrenen, jungen Ärzten beim Erkennen von Infektionen und Einschätzen des Schweregrads helfen und Entscheidungsprozesse (für oder gegen antiinfektive Therapie) abteilungsintern transparent machen. Durch aktives Ein- beziehen des zuständigen mikrobiologischen Labors können auf diese Weise zudem Probeneingang und -aufarbeitung optimiert werden.

Ein Beispiel für die Arbeit eines ABS-Teams im Bereich Qualitätssicherung von „Diagnostik“ („Diagnostic Stewardship“) in der muskuloskeletalen Chirurgie ist die Entwicklung von Algorithmen zur Diagnose von Periprotheseninfektionen. Hierzu gehört die interdisziplinäre Diskussion zu folgenden Punkten:

- Definition der Anzahl der routinemäßig zu entnehmenden Gewebeproben und Blutkulturen bei V.a. periprothetische Infektion

- Definition der Durchführung histopathologischer Diagnostik aus gewonnenen Gewebeproben

- Festlegungen zur Interpretation von Befunden (Abgrenzung von Kontamination vs. Infektion bei mikrobiologisch nachgewiesenen Erregern)

- Umgang mit „nicht eindeutigen“ Befunden (z. B. histologische Hinweise auf Infektion, jedoch kein mikrobiologischer Erregernachweis)

- Diskussion über das Für und Wider alternativer Labordiagnostikverfahren mit dem Mikrobiologen (z. B. Einsenden von explantierten Prothesen oder Sonifikation)

\section{PRAXISTIPP 3}

Das Einbinden von medizinischen Mikrobiologen z. B. in klinikinterne Fortbildungen zum Thema Diagnostik kann Chirurgen dabei unterstützen, bei der Interpretation von Befunden Infektionen sicherer von Kontaminationen abzugrenzen und Kosten für unnötige Diagnostik zu vermeiden.

\section{Antibiotikatherapie}

Zu den Kernstrategien von ABS-Maßnahmen ( $\triangleright$ Tab. 2) gehören sowohl das Erstellen von lokalen Therapieempfehlungen, deren Schulung als auch die Durchführung von regelmäßigen Visiten auf den jeweiligen Stationen.

Das ABS-Team sollte bei der Erstellung lokaler Therapieempfehlungen stets alle weiteren beteiligten Fachdisziplinen, wie z.B. das zuständige mikrobiologische Labor und die Vertreter der Krankenhaushygiene, miteinbeziehen. Dabei ist es essenziell, dass neben der aktuellen klinischen Evidenzlage und aktuellen Leitlinien von Fachgesellschaften auch die jeweilige lokale Resistenzlage und Erregerstatistik beachtet werden und zudem Vorgaben zu Diagnostik (s. o.) von bestimmten Infektionen enthalten sind. Im muskuloskeletalen Bereich bietet sich für ABS-Teams vordringlich die Erarbeitung von Empfehlungen zur Behandlung von Weichteilinfektionen, Infektionen nach Frakturversorgung und periprothetischen In- 
- Tab. 2 Kernelemente von ABS-Arbeit.

Erstellung und Umsetzung von lokalen Behandlungsleitlinien

Definition von lokalen Antiinfektiva-Hauslisten (inkl. Sonderrezeptregelungen, Freigaberegelungen, Anwendungsbeschränkungen)

Gestaltung und Umsetzung von Schulungen, Fortbildungen und Information

Durchführung proaktiver Antiinfektiva-Verordnungsanalysen

Durchführung proaktiver Antiinfektivavisiten

Qualitätsindikatoren: Integration in lokale Qualitätssicherung (Arzneimittelkommission, Hygienekommission); Festlegung und Überprüfung von Qualitätsindikatoren (z. B. Umsetzung PAP), C.-difficile-Inzidenz, Veränderungen der Resistenzstatistik)

ergänzende Strategien: Festlegungen zur Deeskalation, Therapiedauer, Oralisierung, Dosisoptimierung (z. B. TDM), Substanzwechsel/ „Cycling“, selektive mikrobiologische Befundung (selektive Antibiogrammberichtung), Informationstechnologie, Management multiresistenter Erreger

Voraussetzungen: regelmäßige Analyse der lokalen Daten zum Einsatz von Antiinfektiva, der Erregerstatistik (Welche Erreger verursachen Protheseninfektionen?) und der AntiinfektivaResistenzstatistik (Gegen welche Antibiotika sind die häufigsten Erreger von Protheseninfektionen empfindlich?)

fektionen, aber auch von Harnwegsinfektionen und Pneumonien an.

Es bietet sich an, die so erarbeiteten Diagnostik- und Therapieempfehlungen den behandelnden Ärzten sowohl in elektronischer Form als auch als schriftlich (z.B. als Pocket Card oder als Poster im Arztzimmer) zugänglich zu machen. Während zu den Vorteilen einer elektronischen Form insbesondere die schnelle Aktualisierbarkeit (z. B. bei Änderungen von Leitlinien oder bestehenden Lieferengpässen bestimmter Antibiotika) zählen, sind gedruckte Versionen im klinischen Alltag für Chirurgen besonders auf Visiten und bei der Tätigkeit in der Ambulanz schnell verfügbar.

\section{PRAXISTIPP 4}

Das Erstellen lokaler Therapieleitlinien zu bestimmten Infektionen (z. B. zur kalkulierten Behandlung von Haut- und Weichgewebeinfektionen), die allen Ärzten dann als Kitteltaschenkarte zur Verfügung gestellt werden, erleichtert gerade jungen Ärzten die tägliche Arbeit.

Darüber hinaus sollten die für die einzelnen Abteilungen wichtigen Infektionen grundsätzlich auch in Fortbildungsveranstaltungen erläutert werden. Diese bieten die Möglichkeit, die Teilnehmer zu infektiologischen Fragestellungen zu schulen und die Hintergründe für die Behandlungsempfehlungen darzulegen.
Eine Restriktion bestimmter antimikrobieller Substanzen, z. B. durch Forderung einer Rücksprache mit einem Mitglied des ABS-Teams vor Bestellung der Substanz in der Apotheke, kann ebenfalls zur Reduktion einer inadäquaten Antiinfektivatherapie beitragen. Zudem wird diese Maßnahme bei eingeschränkt verfügbaren Antibiotikasubstanzen (Lieferengpässe) immer wichtiger. Dies kann jedoch auch zu besonderen Problematiken führen: Zum einen fehlt es vielerorts an ausreichendem Personal, um rund um die Uhr eine adäquate Bewertung einer Anforderung zu gewährleisten. Zum anderen können Restriktionen einzelner Substanzen zu einem erhöhten Verbrauch alternativer, ebenso unerwünschter Substanzen führen und auf der subjektiven Ebene bei den behandelnden Ärzten den Eindruck einer eingeschränkten Therapiefreiheit erwecken.

Die Umsetzungen dieser Therapieempfehlungen können dann im Rahmen regelmäßiger (z. B. wöchentlicher) „Antibiotikavisiten “ auf den jeweiligen Stationen überprüft und mit den Behandlern besprochen werden („Audit and Feedback"), was auch Teil des kontinuierlichen Schulungskonzepts ist. Üblicherweise wird zunächst die klinische Situation mit dem behandelnden Arzt besprochen und dann diskutiert, ob im konkreten Fall eine antiinfektive Therapie notwendig und eine Optimierung (z.B. Deeskalation, gezielte Erregertherapie) möglich ist.

Im Rahmen dieser ABS-Visiten sollten vor allen Dingen die folgenden Aspekte besprochen werden:

- intraoperativer Befund

- aktuelle Wundsituation

- vorliegende mikrobiologische Diagnostik und ihre Interpretation; Notwendigkeit weiterer Diagnostik

- Möglichkeiten der Deeskalation der antibiotischen Medikation

- Optimierung der Dosierung

- Festlegung der Therapiedauer

- Check von Arzneimittelinteraktionen

\section{PRAXISTIPP 5}

Bereits durch eine kurze Besprechung der bestehenden Antibiotikatherapie (z. B. durch eine einfache Frage wie „Braucht dieser Patient dieses Antibiotikum?" während der täglichen Visite) kann diese bereits verbessert und eine unnötig lange Therapiedauer vermieden werden.

Eine antiinfektive Therapie hat nur dann Aussicht auf Erfolg, wenn von Beginn an die adäquate Dosis appliziert wird. Bei einer optimierten Dosierung ist stets die Vermeidung möglicher Toxizitäten gegen eine mögliche Unterdosierung und ein damit verbundenes hohes Risiko des Therapieversagens und des erhöhten Resistenzdrucks ab- 
zuwägen. In-vitro-Untersuchungen zeigen zudem, dass eine subtherapeutische Antibiotikakonzentration die Bildung von bakteriellen Biofilmen induzieren kann [18].

Bei Glykopeptiden (z. B. Vancomycin) und Aminoglykosiden (Gentamycin) ist aufgrund möglicher Organschädigungen (insbesondere Nephrotoxizität, Ototoxizität) eine Überdosierung zu vermeiden. In der Regel steht für diese Substanzen in der klinischen Routine ein therapeutisches Drug Monitoring (TDM) zur Verfügung, sodass eine engmaschige Kontrolle der Blutkonzentrationen erfolgen kann. Problematischer ist die Situation bei den häufig verwandten $\beta$-Lactam-Antibiotika, für die eine Spiegelmessung nur an wenigen Krankenhäusern verfügbar ist. Eine Reihe von Untersuchungen bei kritisch kranken Patienten hat gezeigt, dass allgemeine Dosierungsschemata auf Grundlage der Zulassungsdaten in vielen Fällen zu Unterdosierung führen [19]. Diese Erkenntnisse legen nahe, dass auch in anderen Bereichen eine Standarddosierung nicht zielführend sein kann.

\section{Merke}

Ein wichtiger Ansatzpunkt von ABS sollte die Implementierung von routinemäßig verfügbarem therapeutischem Drug Monitoring für kritisch zu dosierende Antiinfektiva sein.

Steht ein TDM zur Verfügung, empfiehlt es sich für die Durchführung ebenfalls Standards zu entwickeln und umzusetzen ( $\bullet$ Tab. 3 ).

Die Dokumentation einer antiinfektiven Therapie wird häufig vernachlässigt, gehört aber zu den wichtigen Qualitätsindikatoren [20,21]. Insbesondere in chirurgischen Abteilungen wird ein Patient nicht von einem einzigen Stations- oder Oberarzt betreut, sondern regelmäßig alternierend von unterschiedlichen ärztlichen Kollegen. Nur bei adäquater Dokumentation kann jeder am Behandlungsprozess Beteiligte nachvollziehen, aus welchem Grund welche Therapie für wie lange angesetzt wurde. Hierdurch kann das Risiko einer irrtümlich verlängerten oder auch verkürzten Therapie deutlich reduziert werden. Folglich sollte bei den Visiten nach Möglichkeit stets ein besonderes Augenmerk darauf gelegt werden. So ist es sinnvoll, im gemeinsamen Konsens die angestrebte Dauer der antiinfektiven Therapie bzw. ein Datum für eine Reevaluation (z.B. nach der operativen Fokussanierung) festzulegen und in der Patientenakte zu vermerken.

\section{Merke}

Aus der Patientenakte sollten die folgenden Punkte stets erkennbar sein:

- vorliegende Diagnostik

- Indikation der antiinfektiven Therapie

- Datum des Therapieendes bzw. der Reevaluation der Therapie
- Tab. 3 Beispiel für einen Standard zum therapeutischen Drug Monitoring (TDM) von Vancomycin.

Die Vancomycindosierung erfolgt INITIAL gewichtsadaptiert:

- leichte bis mittelschwere Infektionen: 15-20 mg/kg Körpergewicht q12h

- schwere, lebensbedrohliche Infektionen: 25-30 mg/kg Körpergewicht q12h

Ein TDM durch Bestimmung des Talspiegels und eine Bestimmung der Nierenfunktion durch Messung des Serumkreatinins erfolgt:

auf peripheren Stationen:

Patienten mit normaler Nierenfunktion:

- für die ersten 7-10 Tage der Therapie: alle 2 Tage

- ab dem 7.-10. Tag: täglich

Patienten mit eingeschränkter Nierenfunktion:

- täglich

auf Intensivstationen:

- täglich

Eine individuelle Dosisanpassung erfolgt anhand der gemessenen Talspiegelkonzentration (Blutabnahme unmittelbar vor Verabreichung der nächsten Dosis):

- grundsätzlich sind Talspiegel von $>10 \mu \mathrm{g} / \mathrm{ml}$ anzustreben

- bei schweren Infektionen sind Talspiegel von 15-20 $\mu \mathrm{g} / \mathrm{ml}$ anzustreben

Bei möglichen Rückfragen kann unter den angegebenen Telefonnummern das ABS-Team kontaktiert werden.

Außerhalb der regelmäßigen Visitentermine sollte das ABS-Team für die Behandler grundsätzlich ansprechbar sein und bei Fragen auf die Station kommen. Möglich ist auch eine zusätzliche interdisziplinäre infektiologische Fallkonferenz, in der Therapiekonzepte für einzelne, besonders komplexe Patienten diskutiert werden. Hierbei können auch weitere Mitglieder der Behandlungsteams (wie z.B. medizinische Mikrobiologen, Intensivmediziner und Pathologen) miteinbezogen werden.

\section{PRAXISTIPP 6}

Durch die Zusammenarbeit mit festen Ansprechpartnern, z. B. mit einem klinischen Pharmazeuten des Krankenhauses und einem medizinischen Mikrobiologen des zugehörigen Labors, etabliert sich oft schon in kurzer Zeit ein interdisziplinärer Therapieansatz. Durch das gegenseitige Kennenlernen lassen sich mögliche Vorbehalte schnell abbauen und aufkommende Fragen oft bereits über „den kleinen Dienstweg“ klären.

\section{Zusammenfassung}

Nur durch eine verstärkte interdisziplinäre Zusammenarbeit von Chirurgen, Infektiologen, Apothekern und Mikrobiologen lässt sich in Zeiten steigender Antibiotikaresistenzen Prävention und Therapie von Infektionen langfristig optimieren. ABS stellt ein Bündel von Maßnah- 
men dar, die dabei helfen können, dieses Ziel zu erreichen.

Interessenkonflikt

Die Autoren geben an, dass kein Interessenkonflikt besteht.

Über die Autoren

Dagmar Horn

Apothekerin, ABS-Expertin (DGI), Klinik und Poliklinik für Unfall-, Hand- und Wiederherstellungschirurgie, Universitätsklinikum Münster

\section{Robin Köck}

PD Dr. med., Oberarzt, Mikrobiologe und Hygiene, ABS-Experte (DGI), Institut für Krankenhaushygiene Oldenburg

\section{Dirk Wähnert}

PD Dr. med., Funktionsoberarzt, Unfallchirurgie, Klinik und Poliklinik für Unfall-, Hand- und Wiederherstellungschirurgie, Universitätsklinikum Münster

\section{Steffen Roßlenbroich}

Dr. med., Funktionsoberarzt, Unfallchirurgie, Klinik und Poliklinik für Unfall-, Hand- und Wiederherstellungschirurgie, Universitätsklinikum Münster

\section{Michael J. Raschke}

Univ.-Prof., Klinikdirektor, Unfallchirurgie, Klinik und Poliklinik für Unfall-, Hand- und Wiederherstellungschirurgie, Universitätsklinikum Münster

\section{Korrespondenzadresse}

\section{Dagmar Horn}

Klinik und Poliklinik für Unfall-, Hand- und

Wiederherstellungschirurgie

Universitätsklinikum Münster

Albert-Schweitzer-Campus 1, Gebäude W1

48149 Münster

dagmar.horn@ukmuenster.de

\section{Literatur}

[1] Behnke M, Hansen S, Leistner R et al. Nosocomial infection and antibiotic use: a second national prevalence study in Germany. Dtsch Arztebl Int 2013; 110: 627-633

[2] Renz N, Müller M, Perka C et al. Implantatassoziierte Infektion - Diagnostik. Chirurg 2016; 87: 813-821

[3] Dowsey MM, Choong PFM. Obesity is a major risk factor for prosthetic infection after primary hip arthroplasty. Clin Orthop Relat Res 2008; 466: 153-158

[4] Kampf G, Gastmeier P, Wischnewski $\mathrm{N}$ et al. Analysis of risk factors for nosocomial infections - results from the first national prevalence survey in Germany (NIDEP Study, Part 1). J Hosp Infect 1997; 37: 103-112

[5] Kaye KS, Schmit K, Pieper C et al. The effect of increasing age on the risk of surgical site infection. J Infect Dis 2005; 191: 1056-1062
[6] Berkes M, Obremskey WT, Scannell B et al. Maintenance of hardware after early postoperative infection following fracture internal fixation. J Bone Joint Surg Am 2010; 92: 823-828

[7] Tschudin-Sutter S, Frei R, Dangel $M$ et al. Validation of a treatment algorithm for orthopaedic implant-related infections with device-retention-results from a prospective observational cohort study. Clin Microbiol Infect 2016; 22: 457.e1459.e1

[8] Çakmakçi M. Antibiotic stewardship programmes and the surgeon's role. J Hosp Infect 2015; 89: 264-266

[9] Dik JH, Poelman R, Friedrich AW et al. An integrated stewardship model: antimicrobial, infection prevention and diagnostic (AID). Future Microbiol 2016; 11: 93-102

[10] Nicolau DP. Current challenges in the management of the infected patient. Curr Opin Infect Dis 2011; 24 (Suppl. 1): S1S10

[11] Owens RC jr., Shorr AF, Deschambeault AL. Antimicrobial stewardship: shepherding precious resources. Am J Health Syst Pharm 2009; 66 (Suppl. 4): S15-S22

[12] Hübner C, Hübner N, Muhr M et al. Cost analysis of hospitalized Clostridium difficile-associated diarrhea (CDAD). GMS Hyg Infect Control 2015; 10: Doc13

[13] Kern WV, Fätkenheuer G, Tacconelli E et al. Klinische Infektiologie in Deutschland und Europa. Z Evid Fortbild Qual Gesundhwes 2015; 109: 493-499

[14] Forsblom E, Ruotsalainen E, Ollgren J et al. Telephone consultation cannot replace bedside infectious disease consultation in the management of Staphylococcus aureus bacteremia. Clin Infect Dis 2013; 56: 527-535

[15] Nanchahal J. Standards for the Management of open Fractures of the lower Limb. London: Royal Society of Medicine Press Ltd.; 2009

[16] Hoff WS, Bonadies JA, Cachecho R et al. East Practice Management Guidelines Work Group: update to practice management guidelines for prophylactic antibiotic use in open fractures. J Trauma 2011; 70: 751-754

[17] Horn D, Roßlenbroich S, Köck R, Kreis CAA, Roeder N, Raschke M, Fuchs T. Klinische Pharmazeuten als Teil eines interdisziplinären Teams bei der Antibiotikatherapie in der Unfallchirurgie. Deutscher Kongress für Orthopädie und Unfallchirurgie (DKOU 2015). Berlin, 20.-23.10.2015. Düsseldorf: German Medical Science GMS Publishing House; 2015: DocWI57-1119

[18] Kaplan JB. Antibiotic-induced biofilm formation. Int J Artif Organs 2011; 34: 737-751

[19] Roberts JA, Paul SK, Akova M et al. DALI: defining antibiotic levels in intensive care unit patients: are current $\beta$-lactam antibiotic doses sufficient for critically ill patients? Clin Infect Dis 2014; 58: 1072-1083

[20] Cooke FJ, Matar R, Lawson W et al. Comment on: Antibiotic stewardship - more education and regulation not more availability? J Antimicrob Chemother 2010; 65: 598

[21] Vercheval C, Gillet M, Maes N et al. Quality of documentation on antibiotic therapy in medical records: evaluation of combined interventions in a teaching hospital by repeated point prevalence survey. Eur J Clin Microbiol Infect Dis 2016; 35: 1495-1500

Bibliografie

DOI https://doi.org/10.1055/s-0043-102343

OP-JOURNAL 2017; 33: 178-184 (c) Georg Thieme Verlag KG Stuttgart · New York ISSN 0178-1715 